\title{
Neumonía por Staphylococcus aureus resistente a meticilina adquirido de la comunidad en un hospital pediátrico. Nuestra experiencia de 10 años Community-acquired methicillin-resistant Staphylococcus aureus pneumonia in a children's hospital. Our ten-year experience
}

\author{
Dra. Gabriela Ensinck ${ }^{a}$ Dr. Gustavo Lazarte ${ }^{a}$ Bioq. Adriana Ernst ${ }^{a}$, \\ Dra. Antonela Romagnoli ${ }^{a}$ Dr. Santiago López Papuccia ${ }^{a}$ Dra. Alicia Aletti ${ }^{a}$, \\ Dra. Ana Chiossone ${ }^{a}$, Lic. Fernanda Pigozzi y y Dra. Yanina Sguassero ${ }^{b}$
}

\section{RESUMEN}

Introducción. Las infecciones por Staphylococcus aureus resistente a meticilina adquirido de la comunidad (SARM-AC) se han incrementado en los últimos años. Neumonías necrotizantes y empiemas por SARM-AC son cada vez más frecuentes en niños.

Objetivos. Determinar la prevalencia de neumonías por SARM-AC y sus características clínico-epidemiológicas, en comparación con las neumonías por Streptococcus pneumoniae $(S P)$ en la misma población.

Material y métodos. Estudio descriptivo, observacional, transversal, de pacientes internados con neumonía por SARM-AC en el Hospital de Niños Víctor J. Vilela (período: 1/2008-12/2017).

Resultados. De 54 neumonías por Staphylococcus aureus, 46 (el $85 \%$ ) fueron SARM-AC. El índice deneumonías porSARM-AC varió de 4,9/10 000 (2008) a 10/10 000 egresos (2017). Presentaron sepsis/shock séptico el $41 \%$; empiema, el $96 \%$; neumotórax, el $35 \%$; requirieron drenaje pleural el $90 \%$ y toilette quirúrgica el $55 \%$. Ingresaron a Terapia Intensiva el $65 \%$; la mitad necesitó asistencia respiratoria mecánica. Hubo dos muertes. Resistencia de las cepas: el $17 \%$ a gentamicina, el $13 \%$ a eritromicina, el $11 \%$ a clindamicina. En las neumonías por SARM-AC vs. las neumonías por $S P$, se observó mayor riesgo de sepsis (IC $95 \%$; RR 7,38; 3,32-16,38) e ingreso a Terapia Intensiva (RR 4,29; 2,70-6,83). No hubo muertes por $S P$.

Conclusiones. La prevalencia de neumonías por SARM-AC se duplicó durante la última década. Comparadas con las neumonías por SP, las neumonías por SARM-AC se acompañaron, más frecuentemente, de cuadros de sepsis y shock séptico, ingreso a Terapia Intensiva y asistencia respiratoria.

Palabras clave: neumonía estafilocócica, Staphylococcus aureus resistente a meticilina; estudios transversales; hospitales pediátricos.

http:/ / dx.doi.org/10.5546/ aap.2021.11

Texto completo en inglés:

http:/ / dx.doi.org/10.5546/ aap.2021.eng.11
Cómo citar: Ensinck G, Lazarte G, Ernst A, Romagnoli A, et al. Neumonía por Staphylococcus aureus resistente a meticilina adquirido de la comunidad en un hospital pediátrico. Nuestra experiencia de 10 años. Arch Argent Pediatr 2021;119(1):11-17.

\section{INTRODUCCIÓN}

El incremento de las infecciones por Staphylococcus aureus ( $S A$ ) a nivel mundial ha sido, principalmente, a expensas del Staphylococcus aureus resistente a meticilina adquirido de la comunidad (SARM-AC). ${ }^{1-3}$ Estas cepas poseen un casete cromosómico estafilocócico denominado mec (staphylococcal cassette chromosome mec; SCC mec) tipo IV o V. Este es un elemento genético móvil que lleva el determinante central para la resistencia betalactámica codificada por el gen $m e c A$, que les confiere resistencia a los antibióticos $\beta$-lactámicos y puede acarrear también resistencia acompañante a otros grupos de antibióticos, como macrólidos o aminoglucósidos. . $^{4-6}$ Por lo general, poseen una exotoxina llamada Panton-Valentine leukocidin $(P V L)$, que ha sido relacionada con invasividad, necrosis y mal pronóstico. ${ }^{7.8}$

Las infecciones por SARM-AC se presentan, habitualmente, como infecciones de piel y partes blandas. No obstante, en los últimos años, se ha observado un aumento de las infecciones invasivas, tales como neumonías complicadas, artritis y osteomielitis. ${ }^{9,10}$ Se describe como 
neumonía complicada (NC) aquella que se acompaña de derrame pleural, empiema, neumonía necrotizante o abscedada. ${ }^{11}$

La neumonía estafilocócica era una entidad poco frecuente en pediatría; sin embargo, actualmente, se ha convertido en una patología cada vez más común y con una importante morbimortalidad. ${ }^{12}$ El objetivo principal de nuestro estudio fue evaluar la prevalencia de las neumonías por SARM-AC en la población pediátrica de nuestro Hospital y sus características clínico-epidemiológicas. Como objetivo secundario, se las comparó con la prevalencia y características de las neumonías causadas por Streptococcus pneumoniae (SP) registradas en la misma población durante el período del estudio.

\section{MATERIAL Y MÉTODOS}

Se realizó un estudio descriptivo, prospectivo, de corte transversal en niños y adolescentes con diagnóstico microbiológicamente documentado de neumonías por SARM-AC, evaluados en el Hospital de Niños Víctor J. Vilela durante el período comprendido entre 1/ 2008 y 12/ 2017. Los criterios de exclusión fueron pacientes que, en los seis meses previos al ingreso al estudio, hubiesen permanecido internados (al menos, por 24 horas), visitado un centro para cuidados de salud o estado en contacto con personal de la salud, hubiesen recibido antibióticos o tuvieran el antecedente de aislamiento de un SARM. Se excluyeron, además, los pacientes con comorbilidades al momento del ingreso, tales como inmunosupresión, diabetes, corticoterapia, etc. No se excluyeron pacientes por no contar con todos los datos de las variables analizadas, pero fue aclarado en cada una de ellas. La infección se consideró adquirida en la comunidad cuando se obtuvo desarrollo de $S A$, al menos, en un cultivo de un sitio estéril, tomado de un paciente ambulatorio o dentro de las primeras 48 horas de internación.

En cuanto al diagnóstico microbiológico, la muestra para cultivo se obtuvo de material de punción de sitios estériles (pleural, articular, material quirúrgico, hemocultivos, etc.). El SA se aisló y tipificó mediante pruebas microbiológicas convencionales y automatizadas según los protocolos de trabajo vigentes en el Laboratorio de Microbiología de nuestro Hospital. La sensibilidad a meticilina se determinó por difusión con discos de cefoxitina de 30 microgramos $(\mu \mathrm{g})$; halos menores de 20 milímetros ( $\mathrm{mm}$ ) se consideraron como resistencia. También se determinó la resistencia a rifampicina $(5 \mu \mathrm{g})$, gentamicina $(10 \mu \mathrm{g})$, trimetoprimasulfametoxazol $(25 \mu \mathrm{g})$, eritromicina $(15 \mu \mathrm{g})$, clindamicina $(2 \mu \mathrm{g})$ y ceftarolina $(5 \mu \mathrm{g})$ según las normas internacionales. Los antibiogramas se interpretaron de acuerdo con las normas del Clinical \& Laboratory Standards Institute (CLSI, 2016). ${ }^{13}$ La resistencia inducible a clindamicina se identificó colocando el disco de esta droga a una distancia de $25 \mathrm{~mm}$ del disco de eritromicina en el antibiograma ( $D$ test). La concentración inhibitoria mínima (CIM) a vancomicina se realizó por microdilución o el método de dilución en agar utilizando tiras E-test.

Se diseñó una ficha para la recolección de los siguientes datos: sexo, edad, forma de presentación clínica, tratamiento médico y/o quirúrgico, y sensibilidad antibiótica del microorganismo aislado, duración de la internación, ingreso a Cuidados Intensivos, ingreso a asistencia respiratoria mecánica (ARM) y número de muertes.

El análisis de los datos se realizó de forma global. Se utilizó el programa SAS 9.2 para realizar los análisis estadísticos. Las variables categóricas se describieron como números y porcentajes. Se calculó, además, el riesgo relativo (RR) y el intervalo de confianza (IC) del $95 \%$ para los resultados principales. Las variables continuas se describieron como mediana y rango.

El protocolo de investigación fue avalado por el Comité de Ética de la Secretaría de Salud Pública de la Municipalidad de Rosario y aprobado por el Comité de Docencia e Investigación del Hospital de Niños Víctor J. Vilela. No se solicitó consentimiento informado escrito para el uso de datos. Antes de la inclusión del niño en el estudio, un miembro del equipo de investigación informó a los padres o tutores sobre sus objetivos y el carácter descriptivo, y destacó que la participación no modificaría la conducta clínica ni la indicación de tratamiento del niño. El equipo de investigación garantizó el anonimato y la confidencialidad de los datos.

\section{RESULTADOS}

Durante el período del estudio, 54 pacientes con neumonía por $S A$ fueron elegibles. Se excluyeron 8 por presentar alguno de los criterios de exclusión y se incluyeron y analizaron 46 (el $85 \%$ ). La tasa anual de neumonías por SARMAC respecto a los egresos hospitalarios varió de 4,9/10000 (2008) a 10,1/10000 (2017) (Tabla 1, Figura 1). De los 46 pacientes con neumonías por 
SARM-AC, se observó que la edad promedio fue de 44 meses (mediana de 27,5 meses). Del total, 24 pacientes (el $52 \%$ ) fueron niñas. Los pacientes tuvieron un número de convivientes promedio de 4,5 (rango: 2-8). Se constató el antecedente de infecciones o una infección activa en el momento del diagnóstico en algún miembro de la familia, como impétigo, celulitis, forunculosis o forunculosis recurrente en el 37,5\% de los casos (Tabla 2).

En cuanto a las manifestaciones clínicas, el $41 \%$ presentó un cuadro de sepsis o shock séptico. En la radiografía de tórax, 22/46 (el $48 \%$ ) de los pacientes tuvieron un patrón compatible con neumonía necrotizante o abscedada, y 21/46 (el $46 \%$ ), como neumonía consolidante o lobar. Las complicaciones fueron empiema en el $96 \%$ $(41 / 46)$ y neumotórax en el $35 \%(16 / 46)$. Se acompañaron con manifestaciones cutáneas en 12 pacientes (el $26 \%$ ), seis con exantema de tipo escarlatiniforme y seis con celulitis.

Requirieron tubo de avenamiento pleural el $89 \%$ de los pacientes; 1 tubo: 19 pacientes;
2 tubos: 14 pacientes; y 3 o más tubos: 8 pacientes. El promedio de días de colocación de tubo fue de 16 (rango: 1-63 días). Se realizó toilette quirúrgica en más de la mitad de los pacientes (25/46); en general, se trató de un único procedimiento (en el $75 \%$ ).

La duración total de la internación varió de 1 a 77 días; la mediana fue de 24 días. Los hemocultivos fueron positivos en $10 / 46$ pacientes (el $22 \%$ ). El cultivo de líquido pleural proveniente de 42 muestras mostró una positividad cercana al $90 \%$; el cultivo de biopsia pleural fue positivo en $7 / 11$ pacientes (el $63 \%$ ). Otros cultivos realizados: piel y partes blandas en 5/6, líquido articular en $2 / 3$ y pericárdico en $2 / 4$ fueron positivos.

En el antibiograma, el $17 \%$ de las cepas fueron resistentes a gentamicina; el $13 \%$, a eritromicina; el $11 \%$, a clindamicina, y el $2 \%$, a trimetoprima-sulfametoxazol. No hubo resistencia a teicoplanina, vancomicina, minociclina ni linezolid. La sensibilidad a ceftarolina se realizó en 17 cepas, de las cuales 1 (el 5,7 \%) fue

TABLA 1. Tasa anual de neumonías por Staphylococcus aureus resistente a meticilina adquirido de la comunidad de acuerdo con los egresos hospitalarios. Hospital de Niños Víctor J. Vilela, Rosario. Período 2008-2017

\begin{tabular}{lcccccccccc}
\hline Año & $\mathbf{2 0 0 8}$ & $\mathbf{2 0 0 9}$ & $\mathbf{2 0 1 0}$ & $\mathbf{2 0 1 1}$ & $\mathbf{2 0 1 2}$ & $\mathbf{2 0 1 3}$ & $\mathbf{2 0 1 4}$ & $\mathbf{2 0 1 5}$ & $\mathbf{2 0 1 6}$ & $\mathbf{2 0 1 7}$ \\
\hline Egresos & 8162 & 7323 & 7555 & 7816 & 7773 & 6519 & 7243 & 7380 & 6740 & 6949 \\
$\begin{array}{l}\text { Neumonía por } \\
\text { SARM-AC }\end{array}$ & 4 & 2 & 4 & 3 & 3 & 7 & 6 & 4 & 6 & 7 \\
Tasa por 10000 & 4,9 & 2,7 & 5,3 & 3,8 & 3,9 & 10,7 & 8,3 & 5,4 & 8,9 & 10,1 \\
\hline
\end{tabular}

SARM-AC: Staphylococcus aureus resistente a meticilina adquirido de la comunidad.

Figura 1. Tasa anual de neumonías estafilocócicas por 10000 egresos. Hospital de Niños Víctor J. Vilela. Período 2008-2017

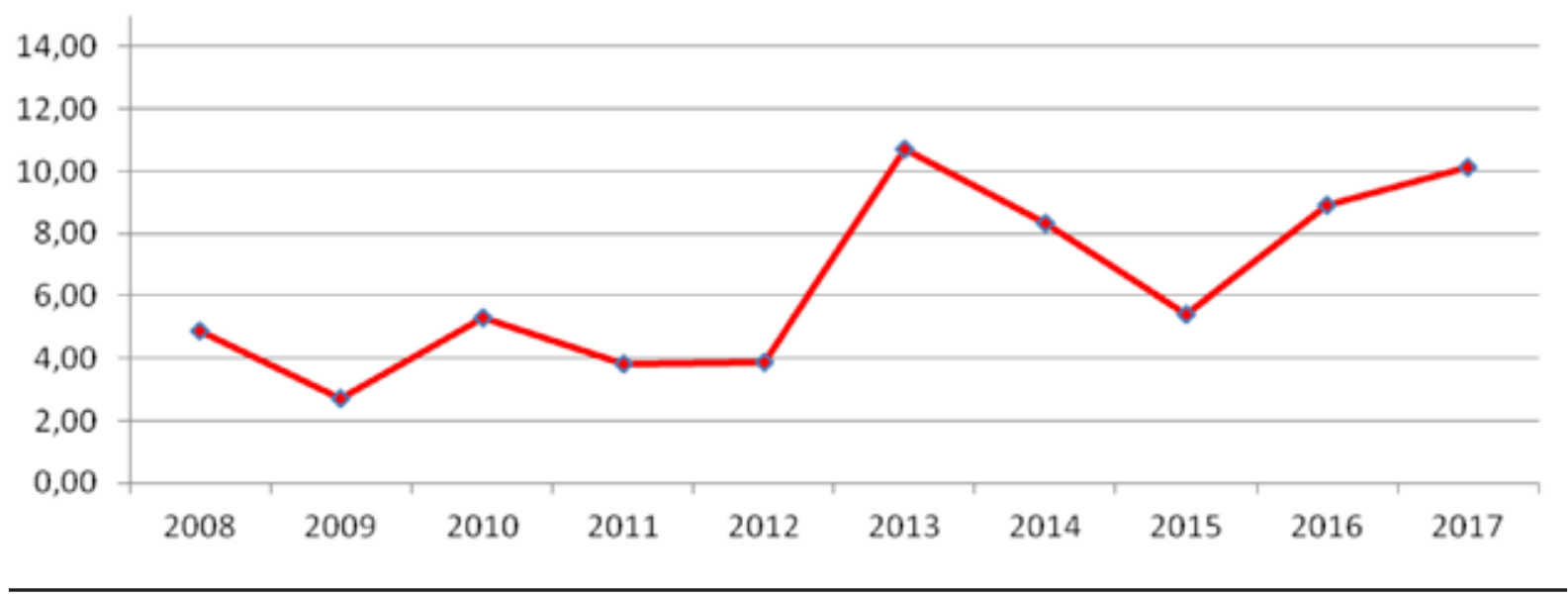


intermedia. La CIM a vancomicina se realizó en 45 muestras; aproximadamente, en el $90 \%$ de las cepas, fue $\leq 1 \mu \mathrm{g} / \mathrm{ml}$ (Tabla 3 ).

En cuanto a la evolución clínica, $30 / 46$ pacientes (el $65 \%$ ) ingresaron a la Unidad de Cuidados Intensivos Pediátricos (UCIP). En este subgrupo, las edades abarcaron de 1 a 132 meses, con una mediana de 23 meses. En promedio, estos pacientes permanecieron internados por 9 días (máximo: 52 días). La mitad de los ingresados a la UCIP $(15 / 30)$ requirieron ARM, con un promedio de estadía de 11,4 días. Dos pacientes (el 4,34\%) de este grupo fallecieron por sepsis y shock séptico.
Cuando se comparan las neumonías por SARMAC con las neumonías por $S P$, se observa un descenso en el número de las neumonías por $S P$ y un ascenso en el de las neumonías por SARMAC (Figura 2) durante los años de estudio. Las neumonías por SARM-AC, respecto a las neumonías por $S P$, tuvieron 7 veces más riesgo de presentarse con cuadros de sepsis o shock séptico, 4 veces más de presentarse como neumonía necrotizante o abscedada y 2 veces más de presentar empiema, 4 veces más de ingresar a la UCIP y 5 veces más de requerir ARM (Tabla 4). Además, a diferencia de la neumonía por SARM-AC, no se registraron muertes en el grupo de las neumonías por SP.

TABla 2. Características clínicas y epidemiológicas de los niños con neumonías por Staphylococcus aureus resistente a meticilina adquirido de la comunidad $(n=46)$ y neumonías neumocócicas $(n=118)$. Hospital de Niños Víctor J. Vilela, Rosario. Período 2008-2017

\begin{tabular}{|c|c|c|c|c|}
\hline VARIABLES & $\begin{array}{l}\text { SARM-AC } \\
n=46\end{array}$ & $\%$ & $\begin{array}{l}\text { S. pneumoniae } \\
\mathbf{n}=\mathbf{1 2 5}\end{array}$ & $\%$ \\
\hline MUJERES & 24 & 52,2 & 56 & 45 \\
\hline \multicolumn{5}{|l|}{ EDAD (MESES) } \\
\hline Promedio & 44 & & 30 & \\
\hline Rango & $1-152$ & & $1-168$ & \\
\hline Mediana & 27,5 & & 25 & \\
\hline \multicolumn{5}{|l|}{ PRESENTACIÓN CLÍNICA* } \\
\hline Neumonía consolidante & 21 & 45,65 & 104 & 83,2 \\
\hline Neumonía necrotizante o abscedada & 22 & 47,8 & 12 & 9,6 \\
\hline Sepsis/shock séptico & 19 & 41,30 & 7 & 5,6 \\
\hline Neumotórax & 16 & 34,78 & 1 & 0,8 \\
\hline Empiema pleural & 44 & 95,6 & 52 & 41,6 \\
\hline Manifestaciones cutáneas & 12 & 26 & 0 & 0 \\
\hline \multicolumn{5}{|l|}{ EVOLUCIÓN } \\
\hline Ingreso a la UCIP & 30 & 65,2 & 19 & 15,2 \\
\hline Edad de los pacientes en la UCIP ${ }^{1}$ & $37,7(1-132)$ & & $31,2(1-119)$ & \\
\hline Promedio de días de internación en la UCIP² & $9,4(1-52)$ & & $6,2(1-22)$ & \\
\hline ARM & 15 & 32,6 & 7 & 5,6 \\
\hline Promedio de días de $\mathrm{ARM}^{2}$ & $11,4(1-43)$ & & $7,7(1-22)$ & \\
\hline Tubos de avenamiento pleural & 41 & 89,13 & 48 & 38,4 \\
\hline Toillete quirúrgica & 25 & 54,3 & 14 & 11,2 \\
\hline
\end{tabular}

* Hubo más de una presentación clínica en el mismo paciente.

${ }^{1}$ Expresado en meses y rango.

${ }^{2}$ Expresado en días y rango.

SARM-AC: Staphylococcus aureus resistente a meticilina adquirido de la comunidad;

UCIP: Unidad de Cuidados Intensivos Pediátricos; ARM: asistencia respiratoria mecánica.

TABLA 3. Concentraciones inhibitorias mínimas a vancomicina de las cepas aisladas de Staphylococcus aureus resistente a meticilina adquirido de la comunidad. Hospital de Niños Víctor J. Vilela de Rosario. Período 2008-2017

\begin{tabular}{lcc}
\hline CIM (ug/ml) & Número & Porcentaje \\
\hline 0,5 & 19 & 42 \\
0,75 & 1 & 2 \\
1 & 20 & 44 \\
1,5 & 5 & 11 \\
\hline
\end{tabular}

CIM: concentraciones inhibitorias mínimas. 


\section{DISCUSIÓN}

En los últimos años, el incremento de las infecciones por SARM-AC ha llevado a un aumento de la consulta médica por este tipo de infección de tres o cuatro veces, y se han observado desde infecciones leves, como las de piel y partes blandas, a otras más invasivas y graves, como sepsis, shock séptico, bacteriemia, infecciones ostearticulares y neumonías complicadas. ${ }^{14,15}$ En una experiencia previa de 10 años en nuestro Hospital, se observó un incremento de las infecciones producidas por SA adquiridas en la comunidad de, aproximadamente, 3 veces, con un promedio de infecciones por SARM-AC del $73 \%$.

En este estudio, la neumonía o empiema producido por SARM-AC fue una de las principales formas de presentación clínica y ocupó el tercer lugar después de las infecciones de piel y partes blandas y las formas ostearticulares. ${ }^{16,17}$ Otras publicaciones internacionales, a su vez, señalan un incremento en la incidencia de las neumonías por SARM-AC, en especial, como neumonías complicadas ${ }^{11,12,18} \mathrm{y}$, principalmente, durante la estación de gripe o influenza. ${ }^{19}$ Este aspecto no fue estudiado en nuestro trabajo.

Durante el período del estudio, la tasa de prevalencia de neumonías producidas por SARMAC se duplicó respecto a los egresos hospitalarios y este incremento fue a expensas, principalmente, del SARM-AC, responsable del $85 \%$ de las cepas. Estudios realizados en la Argentina en 2012-2014 describieron los clones circulantes en la población pediátrica; el más frecuente fue el

Figura 2. Número de casos de neumonías estafilocócicas y neumocócicas por año. Hospital de Niños Víctor J. Vilela. Período 2008-2017

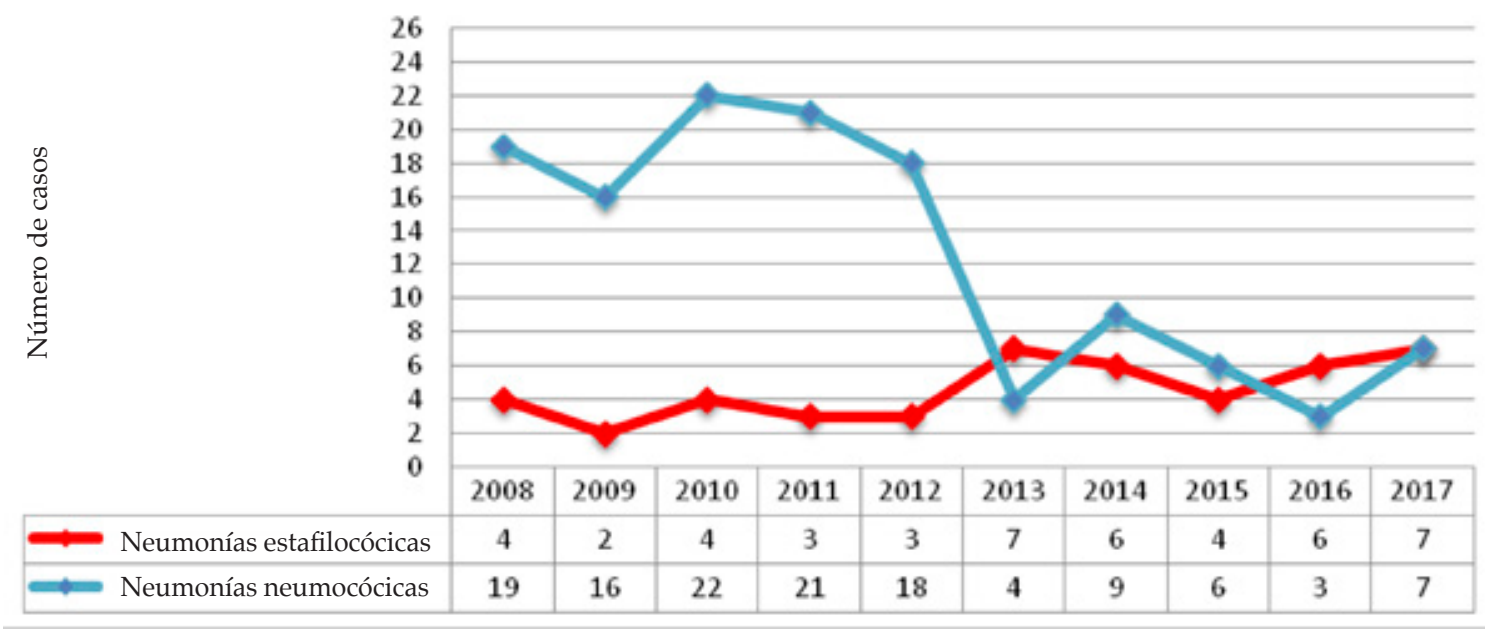

Hospital de Niños Víctor J. Vilela.

TABla 4. Comparación de la forma de presentación y evolución clínica de las neumonías estafilocócicas y neumocócicas. Hospital de Niños Víctor J. Vilela de Rosario. Período 2008-2017

\begin{tabular}{lccc}
\hline & SARM (46) & NEUMOCOCO (125) & RR (IC: 95 \%) \\
\hline Shock o sepsis & 19 & 7 & $7,38(3,32-16,38)$ \\
Supurac. pleuropulmonar & 44 & 52 & $2,30(1,85-2,86)$ \\
Neumonía necrotizante o abscedada & 22 & 12 & $4,98(2,69-9,23)$ \\
Neumotórax & 16 & 1 & $43,48(5,93-318,65)$ \\
Cirugía & 25 & 4 & $4,85(2,77-8,50)$ \\
Tubos & 41 & 48 & $2,14(1,70-2,70)$ \\
UCIP & 30 & 19 & $4,29(2,70-6,83)$ \\
ARM & 15 & 7 & $5,82(2,54-13,37)$ \\
\hline
\end{tabular}

SARM: Staphylococcus aureus resistente a meticilina; RR: riesgo relativo; IC: intervalo de confianza; UCIP: Unidad de Cuidados Intensivos Pediátricos; ARM: asistencia respiratoria mecánica. 
ST5-IV-PVL+. ${ }^{20,21}$ El promedio de edad de los pacientes fue cercano a 4 años; sin embargo, la mediana de edad fue 27,5 meses, datos similares a lo publicado por otros autores. ${ }^{18,22}$

Distintos autores demuestran que el nivel socioeconómico de la población afectada puede influir en la adquisición de estas infecciones, por lo que se relaciona este aspecto con la pobreza y el hacinamiento, como así también con factores biológicos propios del huésped. ${ }^{22,23}$ En este estudio, no se analizó el estado socioeconómico de la población, pero sí el número de convivientes, que fue, en promedio, mayor de 4 personas por domicilio. También se observó que un porcentaje cercano al $40 \%$ de los casos tenía un contacto familiar con el antecedente o una infección presente al momento del diagnóstico. Este fenómeno de transmisión de la infección que, en algún momento, fue descrito en los deportes de roce o en prisioneros ${ }^{3}$ es probable que, en nuestra población, se relacione con condiciones de pobreza, hacinamiento y con dificultades en la higiene personal.

Aproximadamente, el $40 \%$ de los pacientes se presentaron con clínica de sepsis o shock séptico; dos de los pacientes fallecieron con este tipo de cuadros. Esta evolución aguda como neumonías complicadas acompañadas de compromiso sistémico y necesidad de la UCIP y de ARM fue referido con porcentajes similares por otros autores. ${ }^{18,24}$ Diferentes publicaciones postulan que hay correlación entre la presencia de neumonías necrotizantes, abscedadas o empiema y un factor de virulencia del $S A$ conocido como $P V L{ }^{25,26}$ La $P V L$ es una citotoxina que causa destrucción de leucocitos y necrosis de tejidos, lo que, sin duda, facilita a este microorganismo la capacidad de producir abscesos. Esta citotoxina fue encontrada tanto en el $S$. aureus meticilino sensible (SAMS) como en los SARM, por lo cual no se considera la resistencia a meticilina un predictor de gravedad. ${ }^{27} \mathrm{Un}$ metaanálisis publicado en los últimos años avala la asociación de $P V L$ y lesiones de piel y partes blandas, y aleja la asociación de $P V L$ con infecciones invasivas. ${ }^{28}$ Cerca de la mitad de los pacientes presentaron características radiológicas de neumonía necrotizante o abscedada. Es probable que la característica necrotizante de las neumonías por SARM-AC, junto con el requerimiento de ARM, sea una combinación necesaria para la aparición de neumotórax durante la evolución de los cuadros.

El rescate microbiológico fue alto, cercano al $90 \%$ en líquido pleural y mayor del $20 \%$ en hemocultivos. Estas cifras son similares a las publicadas por otros autores. ${ }^{18}$ Dicha situación nos lleva a reflexionar sobre la importancia de la toma de cultivos, sobre todo, la punción pleural diagnóstica, antes de la indicación del tratamiento antibiótico. La resistencia a clindamicina fue inferior a la referida en otras publicaciones ${ }^{24}$ y hubo muy baja resistencia a trimetoprimasulfametoxazol. No se constató la resistencia a teicoplanina, vancomicina, minociclina, linezolid ni ceftarolina, por lo cual estas drogas deben tenerse en cuenta para el tratamiento de dichas infecciones. ${ }^{29}$

Cuando se compararon las neumonías neumocócicas con las estafilocócicas, ambas con aislamiento microbiológico, se observó, en primer lugar, una reducción de las neumonías neumocócicas, con un incremento de las neumonías estafilocócicas. Esta observación, sin duda, está relacionada con la introducción de la vacuna antineumocócica 13-valente a nuestro calendario de vacunación en el año 2011. Un comportamiento similar ha sido reportado por otros autores. ${ }^{1,30}$ Las neumonías estafilocócicas se presentaron en casi la mitad de los casos en forma de sepsis o shock séptico y, más habitualmente, como neumonía necrotizante o empiema, lo que requirió, con mayor frecuencia, UCIP, ARM, toilette quirúrgica y tubos de avenamiento pleural. Cabe destacar que las neumonías por SARM-AC tuvieron 40 veces más riesgo de complicarse con neumotórax, lo que habla de sus características necrotizantes y de los procedimientos que requirieron como parte de su tratamiento. $8,18,19,24$

Dentro de las limitaciones de nuestro estudio, se destaca el hecho de que fue realizado en un centro hospitalario de derivación pediátrica y, por lo tanto, los hallazgos deben ser extrapolados con cautela a otros contextos de atención hospitalaria. Además, el diagnóstico de las neumonías bacterianas sin derrame tiene la dificultad de que los hemocultivos son positivos solo en el 10-20\% de los casos, por lo cual el rescate microbiológico sin la presencia de empiema o derrame pleural suele ser muy bajo. El escaso número de fallecidos no permite evaluar el impacto de esta infección en la mortalidad.

Dentro de las fortalezas de este estudio, es su carácter prospectivo para la recolección planificada de los datos lo que permitió, entre otros aportes para el contexto local, constatar el aumento en la prevalencia de las neumonías por SARM-AC en la última década y delinear 
las características de presentación que hacen al manejo y tratamiento clínico.

\section{CONCLUSIONES}

Del total de neumonías por SA adquiridas en la comunidad, el $85 \%$ fueron SARM-AC. La tasa anual de neumonías por SARM-AC aumentó de 4,9/10 000 a 10,1/10 000 egresos en los últimos 10 años. Comparadas con las neumonías por $S P$, las neumonías por SARM-AC se acompañaron, más frecuentemente, de cuadros de sepsis o shock séptico, necrosis y empiema, y requirieron cinco veces más drenaje quirúrgico, ingreso a la UCIP y ARM.

\section{REFERENCIAS}

1. Buckingham SC, McDougal LK, Cathey LD, Comeaux K, et al. Emergence of community-associated methicillinresistant Staphylococcus aureus at a Memphis, Tennessee Children's Hospital. Pediatr Infect Dis J. 2004; 23(7):619-24.

2. MillerLG,KaplanSL.Staphylococcusaureus: A Community Pathogen. Infect Dis Clin North Am. 2009; 23(1):35-52.

3. KirklandEB,AdamsBB.Methicillin-resistantStaphylococcus aureus and athletes. J Am Acad Dermatol. 2008; 59(3):494502.

4. International Working Group on the Classification of Staphylococcal Cassette Chromosome Elements (IWGSCC). Classification of staphylococcal cassette chromosome mec (SCCmec): Guidelines for reporting novel SCCmec elements. Antimicrob Agents Chemother. 2009;53(12):4961-7.

5. Paganini H, Della Latta M, Muller Opet B, Ezcurra G, et al. Estudio multicéntrico sobre las infecciones pediátricas por Staphylococcus aureus meticilino-resistente provenientes de la comunidad en la Argentina. Arch Argent Pediatr. 2008; 106(5):397-403.

6. Frei CR, Miller ML, Lewis JS 2nd, Lawson KA, et al. Trimethoprim-sulfamethoxazole or clindamycin for community-associated MRSA (CA-MRSA) skin infections. J Am Board Fam Med. 2010; 23(6):714-9.

7. Morgan MS. Diagnosis and treatment of Panton-Valentine leukocidin (PVL)-associated staphylococcal pneumonia. Int J Antimicrob Agents. 2007; 30(4):289-96.

8. Rouzic N, Janvier F, Libert N, Javouhey E, et al. Prompt and successful toxin-targeting treatment of three patients with necrotizing pneumonia due to Staphylococcus aureus strains carrying the panton-valentine leukocidin genes. $J$ Clin Microbiol. 2010; 48(5):1952-5.

9. Ensinck G, Casanueva E, Sguassero Y, Moyano M, et al. Infecciones de piel y partes blandas en pediatría: consenso sobre diagnóstico y tratamiento. Parte 2. Arch Argent Pediatr. 2014; 112(2):183-91.

10. Gentile A, Bakir J, Ensinck G, Cancellara A, et al. Staphylococcus C. meticilino resistente adquirido en la comunidad : hospitalización y riesgo de letalidad en 10 centros pediátricos de Argentina. Arch Argent Pediatr. 2018; 116(1):e47-53.

11. Haji T, Byrne A, Kovesi T. Trends in Pediatric Complicated Pneumonia in an Ontario Local Health Integration Network. Children (Basel). 2018; 5(3):36.

12. Rubinstein E, Kollef MH, Nathwani D. Pneumonia Caused by Methicillin $\square$ Resistant Staphylococcus aureus. Clin Infect Dis. 2008; 46(Suppl 5):S378-85.

13. Clinical and Laboratory Standards Institute (CLSI). Performance standards for antimicrobial susceptibility Testing. 30th ed. CLSI supplement M100. Pennsylvania: CLSI; 2020. [Acceso: 27 de julio de 2020]. Disponible en: http://clsi.org/m100.
14. Wallin TR, Hern HG, Frazee BW. Community-Associated Methicillin-Resistant Staphylococcus aureus. Emerg Med Clin North Am. 2008; 26(2):431-55.

15. Iwamoto M, Mu Y, Lynfield R, Bulens S, et al. Trends in invasive methicillin-resistant Staphylococcus aureus infections. Pediatrics. 2013; 132(4):e817-24.

16. Ensinck G, Ernst A, Lazarte G, Romagnoli A, et al. Infecciones por Staphylococcus aureus meticilino resistente adquirido en la comunidad: experiencia de 10 años en un hospital pediátrico de Rosario, Argentina. Arch Argent Pediatr. 2018; 116(2):119-25.

17. Paganini DH, Verdaguer V, Rodríguez AC, Della Latta $P$, et al. Infecciones causadas por Staphylococcus aureus resistentes a la meticilina en niños provenientes de la comunidad en niños de la Argentina. Arch Argent Pediatr. 2006; 104(4):295-300.

18. Carrillo-Marquez MA, Hulten KG, Hammerman W, Lamberth L, et al. Staphylococcus aureus pneumonia in children in the era of community-acquired methicillinresistance at Texas Children's Hospital. Pediatr Infect Dis J. 2011; 30(7):545-50.

19. LiHT, Zhang TT, Huang J, Zhou YQ, et al. Factors associated with the outcome of life-threatening necrotizing pneumonia due to community-acquired Staphylococcus aureus in adult and adolescent patients. Respiration. 2011; 81(6):448-60.

20. Sola C, Paganini H, Egea AL, Moyano A, et al. Spread of epidemic MRSA-ST5-IV clone encoding PVL as a major cause of community onset staphylococcal infections in Argentinean children. PLoS One. 2012; 7(1):e30487.

21. Egea AL, Gagetti P, Lamberghini R, Faccone D, et al. New patterns of methicillin-resistant Staphylococcus aureus (MRSA) clones, community-associated MRSA genotypes behave like healthcare-associated MRSA genotypes within hospitals, Argentina. Int J Med Microbiol.2014;304(8):108699.

22. See I, Wesson P, Gualandi N, Dumyati G, et al. Socioeconomic factors explain racial disparities in invasive community-associated methicillin-resistant staphylococcus aureus disease rates. Clin Infect Dis. 2017; 64(5):597-604.

23. Leggiadro RJ (ed.). Trends in Invasive Methicillin-resistant Staphylococcus aureus Infections. Current Abstracts. Pediatr Infect Dis J. 2014; 33(3):262.

24. Doudoulakakis AG, Bouras D, Drougka E, Kazantzi M, et al. Community-associated Staphylococcus aureus pneumonia among Greek children: epidemiology, molecular characteristics, treatment, and outcome. Eur J Clin Microbiol Infect Dis. 2016; 35(7):1177-85.

25. Adler A, Temper V, Block CS, Abramson N, et al. PantonValentine leukocidin-producing Staphylococcus aureus. Emerg Infect Dis. 2006; 12(11):1789-90.

26. Tristan A, Bes M, Meugnier H, Lina G, et al. Global distribution of Panton-Valentine leukocidin - positive methicillin-resistant Staphylococcus aureus, 2006. Emerg Infect Dis. 2007; 13(4):594:600.

27. Sicot N, Khanafer N, Meyssonnier V, Dumitrescu O, et al. Methicillin resistance is not a predictor of severity in community -acquired Staphylococcus aureus necrotizing pneumonia- results of a prospective observational study. Clin Microbiol Infect. 2013; 19(3):E142-8.

28. Schallcross LJ, Fragaszy E, Johnson AM, Hayward AC. The role of the Panton-Valentine leucocidin toxin in staphylococcal disease: A systematic review and metaanalysis. Lancet Infect Dis. 2013; 13(1):43-54.

29. Turner NA, Sharma-Kuinkel BK, Maskarinec SA, Eichenberger E, et al. Methicillin-resistant Staphylococcus aureus: an overview of basic and clinical research. Nat Rev Microbiol. 2019; 17(4):203-18.

30. Ves Losada J, Graziano AP, De Abreu M, Blanco M, et al. Infecciones graves por Staphylococcus aureus: características clínicas, sensibilidad antibiótica y uso de antimicrobianos. Serie de casos. Arch Argent Pediatr. 2014; 112(4):e152-5 